\title{
ExaGeoStat: A High Performance Unified Software for Geostatistics on Manycore Systems
}

\author{
Sameh Abdulah, Hatem Ltaief, Ying Sun, Marc G. Genton, and David E. Keyes
}

\begin{abstract}
We present ExaGeoStat, a high performance software for geospatial statistics in climate and environment modeling. In contrast to simulation based on partial differential equations derived from first-principles modeling, ExaGeoStat employs a statistical model based on the evaluation of the Gaussian log-likelihood function, which operates on a large dense covariance matrix. Generated by the parametrizable Matérn covariance function, the resulting matrix is symmetric and positive definite. The computational tasks involved during the evaluation of the Gaussian log-likelihood function become daunting as the number $n$ of geographical locations grows, as $\mathcal{O}\left(n^{2}\right)$ storage and $\mathcal{O}\left(n^{3}\right)$ operations are required. While many approximation methods have been devised from the side of statistical modeling to ameliorate these polynomial complexities, we are interested here in the complementary approach of evaluating the exact algebraic result by exploiting advances in solution algorithms and many-core computer architectures. Using state-of-the-art high performance dense linear algebra libraries associated with various leading edge parallel architectures (Intel KNLs, NVIDIA GPUs, and distributed-memory systems), ExaGeoStat raises the game for statistical applications from climate and environmental science. ExaGeoStat provides a reference evaluation of statistical parameters, with which to assess the validity of the various approaches based on approximation. The software takes a first step in the merger of large-scale data analytics and extreme computing for geospatial statistical applications, to be followed by additional complexity reducing improvements from the solver side that can be implemented under the same interface. Thus, a single uncompromised statistical model can ultimately be executed in a wide variety of emerging exascale environments.
\end{abstract}

\section{Index Terms-MAXIMUM LIKELIHOOD OPTIMIZATION; MATÉRN COVARIANCE FUNCTION; HIGH PERFORMANCE} COMPUTING; CLIMATE/ENVIRONMENT APPLICATIONS; PREDICTION.

\section{INTRODUCTION}

Big data applications and traditional high performanceoriented computing have followed independent paths to the present, but important opportunities now arise that can be addressed by merging the two. As a prominent big data application, geospatial statistics is increasingly performancebound. This paper describes the Exascale GeoStatistics (ExaGeoStat) software, a high-performance, unified software for geostatistics on manycore systems, which targets climate and environment prediction applications using techniques from geospatial statistics. We believe that such a software may play an important role at the intersection of big data and extreme computing by allowing applications with prohibitively large memory footprints to be deployed at the desired scale on modern hardware architectures, exploiting recent software developments in computational linear algebra. ExaGeoStat is intended to bridge the aforementioned gap, attracting the geospatial statistics community to the vast potential of high-performance computing and providing fresh inspiration for algorithm and software developments to the HPC community.

Applications for climate and environmental predictions are among the principal simulation workloads running on today's supercomputer facilities.These applications usually

- King Abdullah University of Science and Technology (KAUST), Extreme Computing Research Center, Computer, Electrical, and Mathematical Sciences and Engineering Division (CEMSE), Thuwal, 23955-6900, Saudi Arabia.

E-mail: Sameh.Abdulah@kaust.edu.sa

Manuscript received M DD, YYYY; revised M DD, YYYY. approximate state variables by relying on numerical models to solve a complex set of partial differential equations, which are based on a combination of first-principles and empirical models tuned by known measurements, on a highly resolved spatial and temporal grid. Then, the large volume of results this method produces is post-processed to estimate the quantities of interest. Such an approach translates the original big data problem into an HPCoriented problem, by relying on PDE solvers to extract performance on the targeted architectures. Instead, ExaGeoStat employs a compute-intensive statistical model based on the evaluation of the Gaussian log-likelihood function, which operates on a large dense covariance matrix. The matrix is generated directly from the application datasets, using the parametrizable Matern covariance function. The resulting covariance matrix is symmetric and positive-definite. The computational tasks involved during the evaluation of the Gaussian log-likelihood function become daunting as the number $n$ of geographical locations grows, as $\mathcal{O}\left(n^{2}\right)$ storage and $\mathcal{O}\left(n^{3}\right)$ operations are required.

ExaGeoStat's primary goal is not to resolve this complexity challenge per se, but to delay its scaling limitation impact, by maximizing the computational power of emerging architectures. The unified software permits to explore the computational limits using state-of-the-art highperformance dense linear algebra libraries by leveraging a single source code to run on various cutting-edge parallel architectures, e.g., Intel Xeon, Intel manycore Xeon Phi Knights Landing chip (KNL), NVIDIA GPU accelerators, and distributed-memory homogeneous systems. To achieve 
this software productivity, we rely on the dense linear algebra library Chameleon [13], which breaks down the tasks of the traditional bulk-synchronous programming model of LAPACK [4] and renders them for an asynchronous task-based programming model. Task-based programming models have received significant attention in computational science and engineering, since they may achieve greater concurrency and mitigate communication overhead, thus presenting a path to the exascale era [1], [8], [18]. Once a numerical algorithm has been expressed in tasks linked by input-output data dependencies, We use the StarPU dynamic runtime system [5] to schedule the various tasks on the underlying hardware resources. The simulation code need only be written once since StarPU allows porting to its supported architectures. ExaGeoStat may thus positively impact the day-to-day simulation work of end users by efficiently implementing the limiting linear algebra operations on large datasets.

To highlight the software contributions and to verify that the model can be applied to geostatistical applications, we design a synthetic dataset generator, which allows us not only to test the software infrastructure, but also to stress the statistical model accordingly. In addition, we experiment using a soil moisture dataset from the Mississippi River basin. Although we focus only on soil moisture, our software is able to analyze other variables that commonly employ the Gaussian log-likelihood function and its flexible Matérn covariance, such as temperature, wind speed, etc. The distillate of this work is two packages that are publicly released as an open-source under BSD 3-Clause license: ExaGeoStat $\mathrm{C}_{\text {library }}{ }^{1}$ and R-wrapper library ${ }^{2}$.

The remainder of the paper is organized as follows. Section 2 states the problem, describes related work, describes the construction of the climate and environment modeling simulation, and shows how to predict missing measurements using this constructed model in which we apply a geostatistical approach to compute large dense covariance matrix. Section 3 highlights our contributions. Section 4 presents a case study from a large geographic region, the Mississippi River basin, and notes the effects of some alternative representations of distance in this context. Section 5 reviews the dense linear algebra libraries. Section 6 outlines the geostatistical algorithm, as implemented in the ExaGeoStat software, and lays out the overall software stack. Performance results and analysis are presented in Section 7, using the synthetic and the real datasets, and we conclude in Section 8.

\section{Problem Statement}

Applications in climate and environmental science often deal with a very large number of measurements regularly or irregularly located across a geographical region. In geostatistics, these data are usually modeled as a realization from a Gaussian spatial random field. Specifically, let $\mathbf{s}_{1}, \ldots, \mathbf{s}_{n}$ denote $n$ spatial locations in $\mathbb{R}^{d}, d \geq 1$, and let $\mathbf{Z}=\left\{Z\left(\mathbf{s}_{1}\right), \ldots, Z\left(\mathbf{s}_{n}\right)\right\}^{\top}$ be a realization of a Gaussian random field $Z(\mathbf{s})$ at those $n$ locations. For simplicity, assume

1. ExaGeoStat is available at https://github.com/ecrc/exageostat 2. ExaGeoStatR is available at https://github.com/ecrc/exageostatr the random field $Z(\mathbf{s})$ has a mean zero and stationary parametric covariance function $C(\mathbf{h} ; \boldsymbol{\theta})=\operatorname{cov}\{Z(\mathbf{s}), Z(\mathbf{s}+\mathbf{h})\}$, where $\mathbf{h} \in \mathbb{R}^{d}$ is a spatial lag vector and $\boldsymbol{\theta} \in \mathbb{R}^{q}$ is an unknown parameter vector of interest. Denote by $\boldsymbol{\Sigma}(\boldsymbol{\theta})$ the covariance matrix with entries $\boldsymbol{\Sigma}_{i j}=C\left(\mathbf{s}_{i}-\mathbf{s}_{j} ; \boldsymbol{\theta}\right)$, $i, j=1, \ldots, n$. The matrix $\boldsymbol{\Sigma}(\boldsymbol{\theta})$ is symmetric and positive definite. Statistical inference about $\boldsymbol{\theta}$ is often based on the Gaussian log-likelihood function:

$$
\ell(\boldsymbol{\theta})=-\frac{n}{2} \log (2 \pi)-\frac{1}{2} \log |\boldsymbol{\Sigma}(\boldsymbol{\theta})|-\frac{1}{2} \mathbf{Z}^{\top} \boldsymbol{\Sigma}(\boldsymbol{\theta})^{-1} \mathbf{Z} .
$$

The maximum likelihood estimator of $\boldsymbol{\theta}$ is the value $\widehat{\boldsymbol{\theta}}$ that maximizes (1). When the sample size of $n$ locations is large and the locations are irregularly spaced, the evaluation of (1) becomes challenging because the linear solver and logdeterminant involving the $n$-by- $n$ dense and unstructured covariance matrix $\boldsymbol{\Sigma}(\boldsymbol{\theta})$ requires $\mathcal{O}\left(n^{3}\right)$ floating-point operations on $\mathcal{O}\left(n^{2}\right)$ memory. For example, assuming a dataset approximately on a grid with $10^{3}$ longitude values and $10^{3}$ latitude values, the total number of locations will be $10^{6}$. Using double-precision floating-point arithmetic, the total required memory footprint is $10^{12} \times 8$ bytes $\sim 8 \mathrm{~TB}$. The corresponding complexity order is $10^{18}$.

\subsection{Related Work}

In recent years, a large amount of research has been devoted to addressing the aforementioned challenge through various approximations; for example, covariance tapering [24], [41], likelihood approximations in both the spatial [44] and spectral [22] domains, latent processes such as Gaussian predictive processes and fixed rank kriging [7], [15], and Gaussian Markov random field approximations [23], [32], [39], [40]; see Sun [45] for a review. Stein [42] showed that covariance tapering sometimes performs even worse than assuming independent blocks in the covariance; Stein [43] discussed the limitations of low rank approximations; and Markov models depend on the measurements locations, which must be aligned on a fine grid with estimations of the missing values [46]. Very recent methods include the nearest-neighbor Gaussian process models [17], multiresolution Gaussian process models [36], equivalent kriging [11], multi-level restricted Gaussian maximum likelihood estimators [12], and hierarchical low rank representations [29]. However, all these methods reduce the computational cost by either approximating the maximum likelihood estimator, or by using approximate models that may or may not allow for exact computations. In this paper, we propose exploring the computational limits of the exact evaluation of the Gaussian log-likelihood function, i.e., Equation (1) with high-performance computing and implementing modern techniques to solve these fundamental computational problems in geostatistics.

\subsection{Matérn Covariance Functions}

To construct the covariance matrix $\boldsymbol{\Sigma}(\boldsymbol{\theta})$ in Equation (1), a valid (positive definite) parametric covariance model is needed. Among the many possible covariance models in the literature, the Matérn family [34] has gained widespread interest in recent years due to its flexibility. The class of 
Matérn covariance functions [28] is widely used in geostatistics and spatial statistics [14], machine learning [10], image analysis, weather forecasting and climate science. Handcock and Stein [28] introduced the Matern form of spatial correlations into statistics as a flexible parametric class where one parameter determines the smoothness of the underlying spatial random field. The history of this family of models can be found in [27]. The Matérn form also naturally describes the correlation among temperature fields that can be explained by simple energy balance climate models [35]. The Matérn class of covariance functions is defined as

$$
C(r ; \boldsymbol{\theta})=\frac{\theta_{1}}{2^{\theta_{3}-1} \Gamma\left(\theta_{3}\right)}\left(\frac{r}{\theta_{2}}\right)^{\theta_{3}} \mathcal{K}_{\theta_{3}}\left(\frac{r}{\theta_{2}}\right),
$$

where $r=\left\|\mathbf{s}-\mathbf{s}^{\prime}\right\|$ is the distance between two spatial locations, $\mathbf{s}$ and $\mathbf{s}^{\prime}$, and $\boldsymbol{\theta}=\left(\theta_{1}, \theta_{2}, \theta_{3}\right)^{\top}$. Here $\theta_{1}>0$ is the variance, $\theta_{2}>0$ is a spatial range parameter that measures how quickly the correlation of the random field decays with distance, and $\theta_{3}>0$ controls the smoothness of the random field, with larger values of $\theta_{3}$ corresponding to smoother fields.

The function $\mathcal{K}_{\theta_{3}}$ denotes the modified Bessel function of the second kind of order $\theta_{3}$. When $\theta_{3}=1 / 2$, the Matern covariance function reduces to the exponential covariance model $C(r ; \boldsymbol{\theta})=\theta_{1} \exp \left(-r / \theta_{2}\right)$, and describes a rough field, whereas when $\theta_{3}=1$, the Matérn covariance function reduces to the Whittle covariance model $C(r ; \boldsymbol{\theta})=\theta_{1}\left(r / \theta_{2}\right) \mathcal{K}_{1}\left(r / \theta_{2}\right)$, and describes a smooth field. The value $\theta_{3}=\infty$ corresponds to a Gaussian covariance model, which describes a very smooth field infinitely meansquare differentiable. Realizations from a random field with Matérn covariance functions are $\left\lfloor\theta_{3}-1\right\rfloor$ times mean-square differentiable. Thus, the parameter $\theta_{3}$ is used to control the degree of smoothness of the random field.

In theory, the three parameters of the Matérn covariance function need to be positive real numbers, but empirical values derived from the empirical covariance of the data can serve as starting values and provide bounds for the optimization. Moreover, the parameter $\theta_{3}$ is rarely found to be larger than 1 or 2 in geophysical applications, as those already correspond to very smooth realizations.

\subsection{Prediction}

The quality of statistical forecasts could be improved by accurately estimating the unknown parameters of a statistical model. With the aid of a given geospatial data and measurements, the constructed statistical model is able to predict missing measurements at new spatial locations.

Assuming unknown measurements vector $\mathbf{Z}_{1}$ with size $m$ and know measurements vector $\mathbf{Z}_{2}$ with size $n$, the prediction problem can be represented as a multivariate normal joint distribution as follows [16], [25]

$$
\left[\begin{array}{l}
\mathbf{Z}_{1} \\
\mathbf{Z}_{2}
\end{array}\right] \sim N_{m+n}\left(\left[\begin{array}{l}
\boldsymbol{\mu}_{1} \\
\boldsymbol{\mu}_{2}
\end{array}\right],\left[\begin{array}{ll}
\boldsymbol{\Sigma}_{11} & \boldsymbol{\Sigma}_{12} \\
\boldsymbol{\Sigma}_{21} & \boldsymbol{\Sigma}_{22}
\end{array}\right]\right)
$$

with $\boldsymbol{\Sigma}_{11} \in \mathbb{R}^{m \times m}, \boldsymbol{\Sigma}_{12} \in \mathbb{R}^{m \times n}, \boldsymbol{\Sigma}_{21} \in \mathbb{R}^{n \times m}$, and $\boldsymbol{\Sigma}_{22} \in$ $\mathbb{R}^{n \times n}$.

The associated conditional distribution can be represented as

$$
\mathbf{Z}_{1} \mid \mathbf{Z}_{2} \sim N_{m}\left(\boldsymbol{\mu}_{1}+\boldsymbol{\Sigma}_{12} \boldsymbol{\Sigma}_{22}^{-1}\left(\mathbf{Z}_{2}-\boldsymbol{\mu}_{2}\right), \boldsymbol{\Sigma}_{11}-\boldsymbol{\Sigma}_{12} \boldsymbol{\Sigma}_{22}^{-1} \boldsymbol{\Sigma}_{21}\right)
$$

Assuming that the known measurements vector $\mathbf{Z}_{2}$ has a zero-mean function (i.e., $\boldsymbol{\mu}_{1}=0$ and $\boldsymbol{\mu}_{2}=0$ ), the unknown measurements vector $\mathbf{Z}_{1}$ can be predicted using [25]

$$
\mathbf{Z}_{1}=\boldsymbol{\Sigma}_{12} \boldsymbol{\Sigma}_{22}^{-1} \mathbf{Z}_{2}
$$

\section{Contributions}

Our contributions can be summarized as follows:

- We introduce ExaGeoStat, a unified software for computational geostatistics that exploits recent developments in dense linear algebra task-based algorithms associated with dynamic runtime systems.

- The ExaGeoStat software we propose is able to estimate the statistical model parameters for geostatistics applications and predict missing measurements.

- ExaGeoStat relies on a single source code to target various hardware resources including shared and distributed-memory systems composed of contemporary devices, such as traditional Intel multicore processors, Intel manycore processors, and NVIDIA GPU accelerators. This eases the process of software deployment and effectively employs the highly concurrent underlying hardware, thanks to the finegrained, tile-oriented parallelism and dynamic runtime scheduling.

- We propose a synthetic dataset generator that can be used to perform broader scientific experiments related to computational geostatistics applications.

- We propose an R-wrapper functions for the proposed software (i.e., ExaGeoStatR) to facilitate the use of our software in the $R$ environment [31].

- We evaluate the performance of our proposed software during applications using both synthetic and real datasets in terms of elapsed time and number of floating-point operations (Gflop/s) on several hardware systems.

- We assess the quality of the estimation of the Matérn covariance parameters and prediction operation achieved by ExaGeoStat through a quantitative performance analysis and using both exact and approximation techniques.

\section{Climate and Environment Data}

In climate and environment studies, numerical models play an important role in improving our knowledge of the characteristics of the climate system, and of the causes of climate variations. These numerical models describe the evolution of many variables, for example, temperature, wind speed, precipitation, humidity and pressure, by solving a set of equations. The process involves physical parameterization, initial condition configuration, numerical integration, and data output. In this section, we use the proposed methodology to investigate the spatial variability of soil moisture data generated by numerical models. Soil moisture is a key factor in evaluating the state of the hydrological process, and has a 


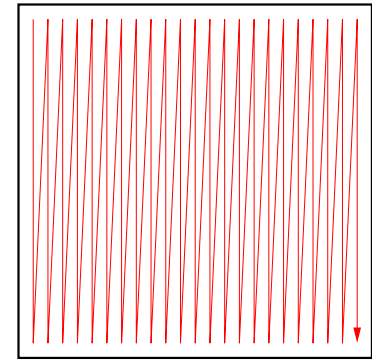

(a) LAPACK: Column-major data layout format.

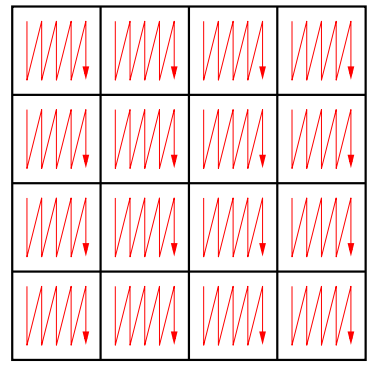

(b) Chameleon: Tile data layout format.
Fig. 1: Data layout format.

wide range of applications in weather forecasting, crop yield prediction, and early warning of flood and drought. It has been shown that better characterization of soil moisture can significantly improve the weather forecasting. However, the numerical models often generate very large datasets due to the high spatial resolutions, which makes the computation of the widely used Gaussian process models infeasible. Consequently, practitioners divide the whole region to smaller size of blocks, and fit Gaussian process models independently to each block, or reduce the size of the dataset by averaging to a lower spatial resolution. However, compared to fitting a consistent Gaussian process model to the entire region, it is unclear how much statistical efficiency is lost by such an approximation. Since our proposed technique can handle large covariance matrix computations, and the parallel implementation of the algorithm significantly reduces the computational time, we propose to use exact maximum likelihood inference for a set of selected regions in the domain of interest to characterize and compare the spatial variabilities of the soil moisture.

We consider high-resolution daily soil moisture data at the top layer of the Mississippi River basin, U.S.A., on January 1st, 2004. The spatial resolution is of 0.0083 degrees, and the distance of one-degree difference in this region is approximately $87.5 \mathrm{~km}$. The grid consists of $1830 \times 1329=2,432,070$ locations with $2,153,888$ measurements and 278,182 missing values. We use the same model for the mean process as in Huang [29], and fit a zero-mean Gaussian process model with a Matérn covariance function to the residuals; see Huang [29] for more details on data description and exploratory data analysis.

\section{State-Of-The-Art Dense Linear Algebra LIBRARIES}

This section recalls the latest developments in dense linear algebra software libraries and their relevant implications.

\subsection{Block Algorithms}

The default paradigm behind LAPACK [4], the wellestablished open-source dense linear algebra library for shared-memory systems, is block-column algorithms. These algorithms decompose the matrix into successive panel and update computational phases, while the matrix is organized

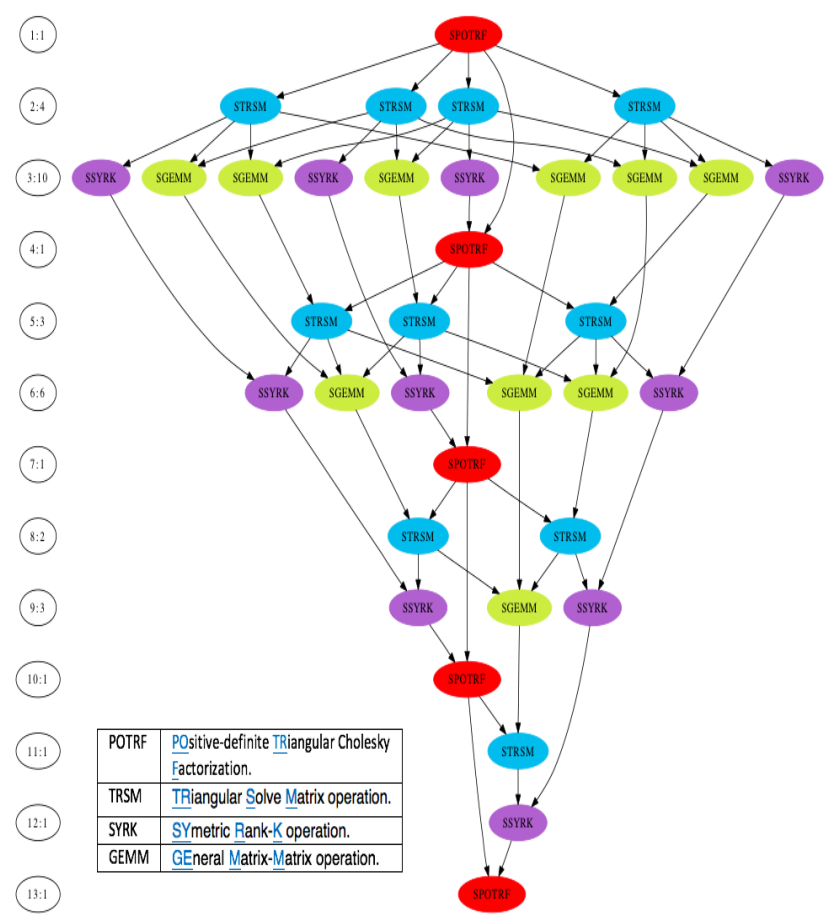

Fig. 2: Directed Acyclic Graph (DAG) for a Cholesky factorization: DAG height corresponds to the length of the critical path and the DAG width to the degree of concurrency.

in a column-major format, see Figure 1(a). The matrix transformations are blocked within the panel factorization phase, and applied together at one time during the update phase. The former is typically memory-bound due to the Level2 BLAS operations, while the latter is compute-intensive due to the Level-3 BLAS updates occurring on the trailing submatrix. LAPACK uses the fork-join paradigm, which has demonstrated scalability issues on multicore architectures. Its distributed version, ScaLAPACK [9] follows the same paradigm and scatters the matrix using a two-dimensional block-cyclic data distribution across a grid of processors to reduce load imbalance and communication overheads.

\subsection{Tile Algorithms}

The tile algorithm methodology [2], [13] splits the matrix into small tiles instead of tall panels, as seen in Figure 1(b), so that updates of the trailing submatrix may be triggered before the current panel factorization is complete. This fine-grained lookahead method exploits more concurrency and enables the maximization of hardware resources by removing synchronization points between the panel and update computational phases. The numerical algorithm can then be translated into a Directed Acyclic Graph (DAG), where the nodes represent tasks and the edges define data dependencies, as highlighted in Figure 2.

\subsection{Dynamic Runtime Systems}

Once the tasks are defined with their respective data dependencies, a dynamic runtime system [6], [20], [21] may be employed directly on the sequential code to schedule 
the various tasks across the underlying hardware resources. Its role is to ensure that the data dependencies are not violated. These runtimes enhance the software productivity by abstracting the hardware complexity from the end users. They are also capable of reducing load imbalance, mitigating data movement overhead, and increasing occupancy on the hardware.

\section{The ExaGeostat Software}

\subsection{General Description}

We propose a unified computational software for geostatistical climate and environmental applications based on the maximum likelihood approach. Since the covariance matrix is symmetric and positive-definite, the computation of the maximum likelihood consists of the Cholesky factorization and its corresponding solver which uses measurements vector $\mathbf{Z}$ as the right-hand side. The log-determinant is calculated from the Cholesky factor simply by computing the product of the diagonal entries.

The objective of this software is not only to solve the maximum likelihood problem for a given set of real measurements, $\mathbf{Z}$, on $n$ geographic locations, but also to predict a set of unknown measurements at new locations. The proposed software also provides a generic tool for generating a reference set of synthetic measurements and locations for statisticians, which generates test cases of prescribed size for standardizing comparisons with other methods.

Our proposed software has two different execution modes for dealing with synthetic and real datasets. In testing mode, ExaGeoStat generates the measurements data based on a given vector $\boldsymbol{\theta}=\left(\theta_{1}, \theta_{2}, \theta_{3}\right)^{\top}$, where $\theta_{1}$ is the variance parameter, $\theta_{2}$ is the range parameter, and $\theta_{3}$ is the smoothness parameter. In this case, the resulting $\hat{\boldsymbol{\theta}}$ vector, which maximizes the likelihood function, should contain a set of values close to the initial $\boldsymbol{\theta}$ vector. Moreover, testing the prediction accuracy can be done by choosing random measurements from the given synthetic dataset and use the generated model to predict these measurements using the other known measurements. The accuracy of the predictions can be verified by comparing random measurements from the given synthetic dataset to the corresponding generated measurements from the model.

In application mode, both the measurements and the locations data are given, so the software is only used to evaluate the MLE function by estimating the parameter vector, $\widehat{\boldsymbol{\theta}}$. The generated model can be used to predict unknown measurements at a set of new locations.

\subsection{Software Infrastructure}

ExaGeoStat internally relies on Chameleon, a high performance numerical library [13]. Based on a tile algorithm, Chameleon is a dense linear algebra library that provides high-performance solvers. Chameleon handles dense linear algebra operations through a sequential task-based algorithms. It features a backend with links to several runtime systems, and in particular, the StarPU dynamic runtime system, which is preferred for its wide hardware architecture support (Intel manycore, NVIDIA GPU, and distributedmemory systems).

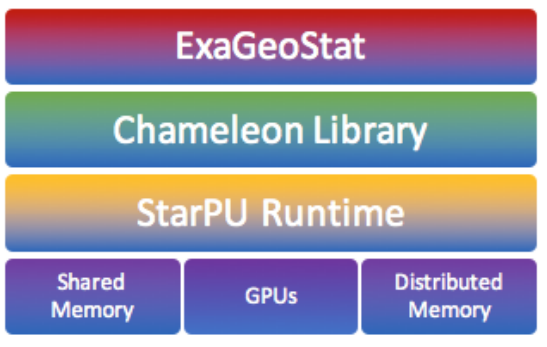

Fig. 3: ExaGeoStat software.

StarPU deals with the execution of generic task graphs, which are generated by a sequential task flow (STF) programming model. The tasks are sequentially given to StarPU with hints of the data dependencies (e.g., read, write, and read-write). The StarPU runtime schedules the given tasks based on these hints. The main advantage of using a runtime system that relies on task-based implementations such as StarPU is to become oblivious of the targeted hardware architecture. This kind of abstraction improves both the user productivity and creativity. Multiple implementations of the same StarPU tasks are generated for: CPU, CUDA, OpenCL, OpenMP, MPI, etc. At runtime, StarPU decides automatically which implementation will achieve the highest performance. For the first execution, StarPU generates a set of cost models that determine best hardware for optimal performance during the given tasks. This set of cost models may be saved for future executions.

Figure 3 shows the structure of the ExaGeoStat software. It has three main layers: ExaGeoStat, which includes the upper-level functions of the software; the Chameleon library, which provides solvers for the linear algebra operations; and the StarPU runtime, which translates the software for execution on the appropriate underlying hardware.

\subsection{The Optimization Framework}

Finding the parameter vector $\widehat{\boldsymbol{\theta}}=\left(\theta_{1}, \ldots, \theta_{q}\right)^{\top}$, that maximizes the likelihood function requires several iterations of the log-likelihood evaluation. In our proposed software, we rely on an open-source $\mathrm{C} / \mathrm{C}++$ nonlinear optimization toolbox, NLopt [30], to perform the optimization task. The NLopt package contains 20 global and local optimization algorithms. NLopt solves nonlinear optimization problems of the form $\min _{\mathbf{x} \in \mathbb{R}^{q}} f(\mathbf{x})$, where $f$ represents the objective function and $\mathbf{x}$ represents the $q$ optimization parameters, i.e., the parameter vector. Because we are targeting a nonlinear problem with a global maximum point, we selected BOBYQA for our proposed platform.

BOBYQA is one of the optimization algorithms available in the sequential Nlopt package to optimize the MLE function. It is a numeric, global, derivative-free and boundconstrained optimization algorithm. It generates a new computed point on each iteration by solving a trust region subproblem subject to given constraints [37], in our case, only upper and lower bound constraints are used. Though BOBYQA does not require the evaluation of the derivatives of the cost function, it employs an iteratively updated quadratic model of the objective, so there is an implicit assumption of smoothness. 
The master process feeds the optimization black box BOBYQA function with the current $\boldsymbol{\theta}$ vector, which produces the resulting likelihood value. This likelihood value gets broadcasted to all other running processes, which, in return, carry on with subsequent computations. This optimization step is then repeated with a new parameter vector $\boldsymbol{\theta}$ at each iteration, until convergence is reached.

As with the linear algebra software, we employ these optimization frameworks without novel contributions herein, in order to achieve the practical synthesis of wellunderstood components. For now, we merely design the interfaces of these codes, which, in the case of BOBYQA, consists mainly of callbacks to the log-likelihood function with a sequence of Matérn triples that must be evaluated using the measurement vector and the covariance matrix. The log-likelihood function may need to be evaluated many times, but after the initial factorization the cost of each estimation step should remain constant.

\subsection{Synthetic Data Generator}

ExaGeoStat provides an internal data generator that is used here to demonstrate the accuracy of the software. This data generator can also be used as a stand-alone tool to generate sets of guided synthetic data for experiments with specific needs or conditions.

Given $n$ locations that are uniformly but randomly distributed, the covariance matrix $\boldsymbol{\Sigma}$ can be built using the Matérn covariance function (i.e., Equation (2)). This covariance matrix can be used to generate a measurement vector $\mathbf{Z}$ from normal variates at the generated $n$ locations, as follows

$\boldsymbol{\Sigma}=\mathbf{L} \cdot \mathbf{L}^{\top} \quad \Rightarrow$ Cholesky factorization.

$\mathbf{Z}=\mathbf{L} \cdot \mathbf{e} \quad \Rightarrow$ where $e_{i} \sim N(0,1)$.

The data generator tool is shown in Algorithm 1. To generate a synthetic measurement vector $\mathbf{Z}$, the algorithm randomly generates a set of $n$ locations (line 2). Then, the distance matrix $\mathbf{D}$ is generated between these $n$ random locations (line 3). In line 4 , an initial covariance matrix $\boldsymbol{\Sigma}$ is generated using the $\mathbf{D}$ matrix and the initial parameter vector $\boldsymbol{\theta}$. In line 5 , a Cholesky factorization step is performed on the covariance matrix $\boldsymbol{\Sigma}$ by using the Chameleon routine dpotrf to generate the lower triangular matrix $\mathbf{L}$. After generating the initial normal random vector, $\mathbf{e}$, a single matrix-vector multiplication operation is performed using the lower triangular matrix $\mathbf{L}$ and the random vector $\mathbf{e}$ to initiate the synthetic measurement vector $\mathbf{Z}$ (lines 6-7). Here, the Chameleon routine $\mathbf{d t r m m}$ is used.

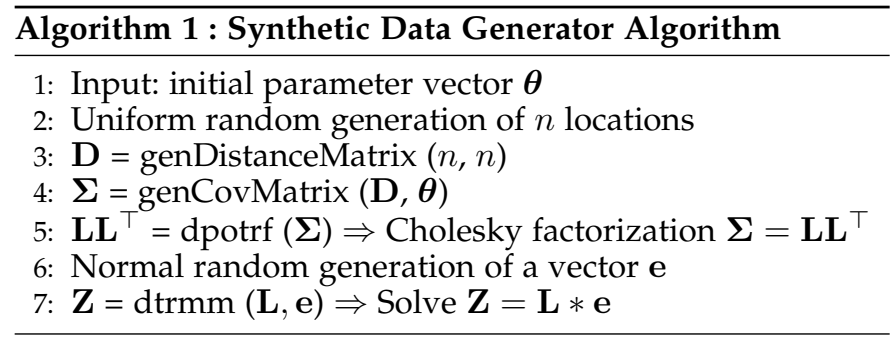

\subsection{Likelihood Evaluation}

As mentioned, our software has two different running modes: testing mode to build a statistical model based on a given set of parameters with the aid of a synthetic set of data (i.e., measurements and locations) and application mode where measurements and locations data are given to estimate the statistical model's parameters for future prediction of unknown measurements at a new set of locations.

For both modes, with a given measurement vector $\mathbf{Z}$ and distance matrix $\mathbf{D}$, the likelihood function can be evaluated using a set of routines from the Chameleon library. The evaluation algorithm based on Equation (1) is presented in detail in Algorithm 2. The inputs to the evaluation algorithm are the measurement vector $\mathbf{Z}$, distance matrix $\mathbf{D}$, and parameter vector $\boldsymbol{\theta}$ (line 1). The algorithm generates the covariance matrix $\boldsymbol{\Sigma}$ (line 2) using the Matérn function given by Equation (2). In line 3, a Cholesky factorization step is performed on the covariance matrix $\boldsymbol{\Sigma}$ by using the dpotrf routine to generate the lower triangular matrix $\mathbf{L}$. In line 4 , a triangular solver $\mathbf{d t r s m}$ is used to solve $\mathbf{L} \times \mathbf{Z}_{\text {new }}=\mathbf{Z}_{\text {old }}$. Both the log-determinant and dot product operations are performed in lines 5-6. In line 7, the likelihood value $\ell$, which should be maximized, is calculated based on the dotscalar and logscalar values.

To find the maximum likelihood value, this algorithm is called several times with different parameter vectors $\boldsymbol{\theta}$ with the help of the used optimization function.

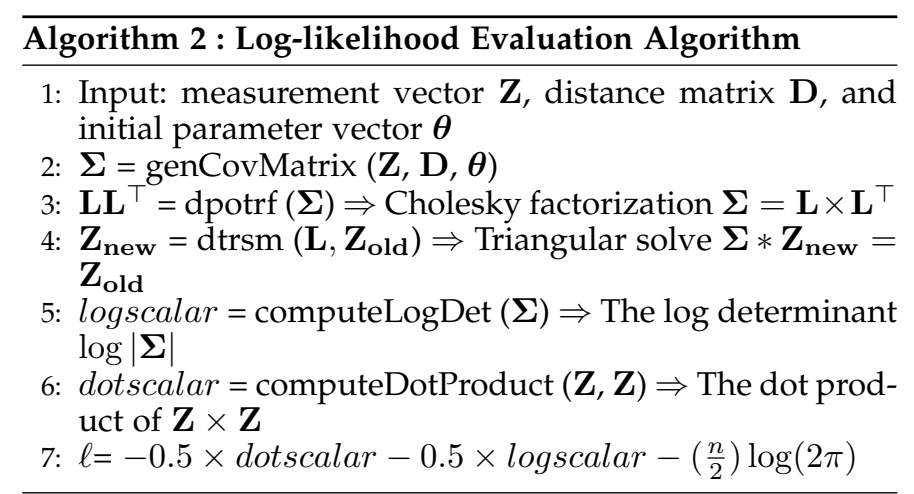

The main goal of Algorithm 2 is to calculate the likelihood function using a certain $\boldsymbol{\theta}$ vector. However, our statistical model relies on finding the parameter vector $\widehat{\boldsymbol{\theta}}$, which maximizes the value of the likelihood function $\ell$. Thus, BOBYQA optimization algorithm is used with the $\theta$ vector and the $\ell$ value to find the optimized vector $\widehat{\boldsymbol{\theta}}$ for the given problem $(\mathbf{Z}, \boldsymbol{\Sigma})$. It is difficult to determine in advance the average number of iterations needed to maximize the likelihood function because it depends on several factors, such as the optimization algorithm, the initial parameters $\boldsymbol{\theta}$, and the maximum acceptable relative tolerance (i.e., the measure of error between the current solution and the previous solution).

\subsection{Prediction}

In the likelihood estimation step, we aim to construct a statistical model based on estimated parameters (i.e., $\widehat{\boldsymbol{\theta}}$ vector). This model can be used for predicting $m$ unknown 
measurement in the vector $\mathbf{Z}_{1}$ with the aid of $n$ known measurement in the vector $\mathbf{Z}_{2}$ (see Equation (5)). The prediction operation can also be implemented using a set of routines from the Chameleon library.

Algorithm 3 shows the prediction algorithm in details. The algorithm has a set of inputs: the parameter vector $\hat{\theta}$, the measurement vector $\mathbf{Z}_{2}$, a vector of the known $n$ locations, and a vector of the new $m$ locations with unknown measurement vector $\mathbf{Z}_{1}$ (line 1). The algorithm aims to predict the measurement vector $\mathbf{Z}_{1}$ at the given $m$ locations (line 2). In lines 3 and 4 , two distance matrices are generated: $\mathbf{D}_{22}$ between two sets of the observed $n$ locations, and $\mathbf{D}_{12}$ between the given unobserved $m$ locations and the observed $n$ locations. These distance matrices are used to construct two covariance matrices, $\boldsymbol{\Sigma}_{\mathbf{2 2}}$ and $\boldsymbol{\Sigma}_{12}$ (lines 5-6). In line 7 , the dposv routine is used to solve the system of linear equation $\mathbf{Z} \times \mathbf{X}=\mathbf{\Sigma}_{22}$. In line 8, the unknown measurement vector, $\mathbf{Z}_{1}$, can be calculated using the dgemm routine (i.e., matrix-matrix multiplication), $\mathbf{Z}_{1}=\mathbf{\Sigma}_{12} \times \mathbf{X}$.

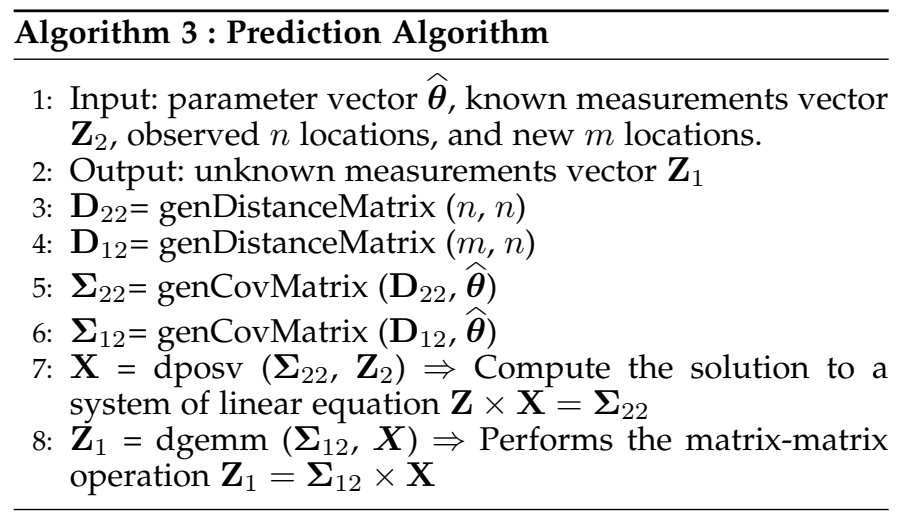

\subsection{Facilitating ExaGeoStat Adoption for Statisticians}

Statisticians rely heavily on $R$, a high productivity simulation environment, to rapidly deploy and assess their algorithms, especially when applied to big data problems, as in climate and environmental research studies. Therefore, we provide $R$-wrappers to our main computational functions through a separate package called ExaGeoStatR. These $\mathrm{R}$ functions should help in disseminating our software toward a large computational statistician community. To the best of our knowledge, most of existing $R$ solutions for the MLE problem are sequential and restricted to limited data sizes such as fields package provided by the University Corporation for Atmospheric Research (UCAR) [19].

\subsection{Independent Blocks (IND) Approximation}

Approximation means exist to reduce the algorithmic complexity when dealing with very large and irregularly spaced geospatial data. Several previous studies show how the likelihood estimation problem can be adapted to provide different methods of hierarchical low-rank approximations. These methods have shown their effectiveness in both computation and accuracy [17], [29], [33], [36], [42], [46].

As mentioned in the introduction section, this paper is mostly focusing on the exact computation of large geospatial

\begin{tabular}{|l|l|l|l|l|l|l|l|}
\hline & & 0 & 0 & 0 & 0 & 0 & 0 \\
\hline & & 0 & 0 & 0 & 0 & 0 & 0 \\
\hline 0 & 0 & & & 0 & 0 & 0 & 0 \\
\hline 0 & 0 & & & 0 & 0 & 0 & 0 \\
\hline 0 & 0 & 0 & 0 & & & 0 & 0 \\
\hline 0 & 0 & 0 & 0 & & & 0 & 0 \\
\hline 0 & 0 & 0 & 0 & 0 & 0 & & \\
\hline 0 & 0 & 0 & 0 & 0 & 0 & & \\
\hline
\end{tabular}

Fig. 4: An example of Independent Blocks (IND) approximation technique on a diagonal 2-by-2 super tile matrix.

data. The quality of exact computation may be demonstrated by comparing it with traditional approximation approaches on the same problem. Thus, in this section, we highlight one of commonly used approximation strategies, i.e., Independent blocks (IND). Such a strategy has been used in different studies and turns out to be a suitable way to reduce the complexity of evaluating the likelihood function on large-scale datasets [29], [42].

The IND approximation technique proceeds by annihilating off-diagonal tiles, since their contributions as well as their qualitative impact on the overall statistical problem may be limited. The implementation of this approximation technique maps well with the inherent tile algorithms of ExaGeoStat and enables to expose tuning parameters, which trades off performance and statistical efficiency. One of these tunable parameters is the size of the diagonal super tile, which determines how many tiles, around the diagonal, need to be aggregated together. A large diagonal super tile basically integrates more statistical contributions from the original problem at the expense of performing more operations. Figure 4 shows an example of the IND approximation technique using a diagonal 2-by-2 super tile matrix.

The IND approximation technique generates a matrix where elements from off-diagonal tiles are set to zero. In this case, applying Cholesky factorization to the whole matrix is unnecessary and time-consuming. Thus, we propose a modified version of the well-known tile Cholesky factorization algorithm presented in [2]. The modified version is aware of the new sparse structure of the matrix and avoids zerostiles during the computation, which speeds-up the Cholesky factorization operation for the whole matrix.

\section{EXPERIMENTAL RESULTS}

\subsection{Environment Settings}

We evaluate the performance of the proposed software on a wide range of manycore-based systems: a dual-socket 28core Intel Skylake Intel Xeon Platinum 8176 CPU running at $2.10 \mathrm{GHz}$, a dual-socket 18-core Intel Haswell Intel Xeon CPU E5-2698 v3 running at $2.30 \mathrm{GHz}$ and equipped with 8 NVIDIA K80s (2 GPUs per board), a dual-socket 14-core Intel Broadwell Intel Xeon E5-2680 V4 running at $2.4 \mathrm{GHz}$, Intel manycore Knights Landing (KNL) 7210 chips with 64 cores, a dual-socket 8-core Intel Sandy Bridge Intel Xeon CPU E5-2650 running at $2.00 \mathrm{GHz}$, and a dual-socket Intel IvyBridge Intel Xeon CPU E5-2680 running at $2.80 \mathrm{GHz}$. 


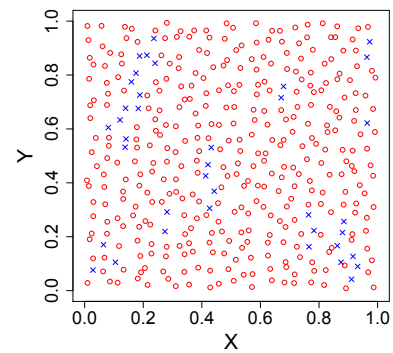

Fig. 5: An example of 400 points irregularly distributed in space, with 362 points ( $\circ$ ) for maximum likelihood estimation and 38 points $(\times)$ for prediction validation.

For the distributed memory experiments, we use KAUST's Cray XC40 system, Shaheen, with 6,174 dualsocket compute nodes based on 16-core Intel Haswell processors running at $2.3 \mathrm{GHz}$, where each node has $128 \mathrm{~GB}$ of DDR4 memory. The Shaheen system has a total of 197,568 processor cores and $790 \mathrm{~TB}$ of aggregate memory.

Our software is compiled with gcc v4.8 and linked against the Chameleon library v0.9.1 with HWLOC v1.11.5, StarPU v1.2.1, Intel MKL v11.3.1, and NLopt v2.4.2 optimization libraries. The LAPACK implementation is the multithreaded version from the vendor optimized Intel MKL v11.3.1 numerical library, available on each platform.

In this study, the synthetic datasets are generated at irregular locations in a two-dimensional space with an unstructured covariance matrix [29], [46]. To ensure that no two locations are too close, the data locations are generated using $n^{1 / 2}\left(r-0.5+X_{r l}, l-0.5+Y_{r l}\right)$ for $r, l \in\left\{1, \ldots, n^{1 / 2}\right\}$, where $n$ represents the number of locations, and $X_{r l}$ and $Y_{r l}$ are generated using uniform distribution on $(-0.4,0.4)$. Figure 5 shows a drawable example of 400 irregularly spaced grid locations in a square region. We only use such a small example to highlight our methodology to generate geospatial data, however, this work uses synthetic datasets up to $49 \times 10^{10}$ locations (i.e., $700 \mathrm{~K} \times 700 \mathrm{~K}$ ).

\subsection{Quantitative Results Assessment}

\subsubsection{Likelihood Estimation Performance}

The first set of experiments highlights the execution time for a single iteration of the MLE algorithm on different target systems. We compare our software with the numerical library LAPACK [4]. LAPACK is considered the main backbone of existing MLE implementations for geostatistical applications [26].

We report the results of several experiments on different hardware architectures: shared-memory, GPUs, and distributed-memory. As the maximum likelihood estimation problem includes an optimization operation with several likelihood estimation iterations, we report only the time to finish one iteration of the likelihood estimation. The LAPACK curves represent the performance of the LAPACKbased ExaGeoStat using all threads. The figures show that the Chameleon-based ExaGeoStat outperforms the LAPACK variant on different platforms when using the same number

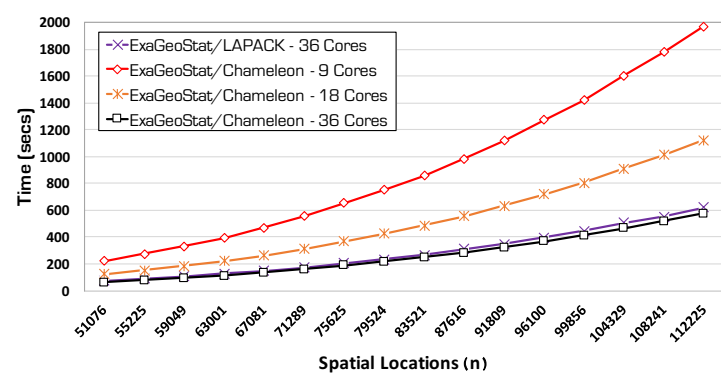

(a) Intel two-socket Haswell.

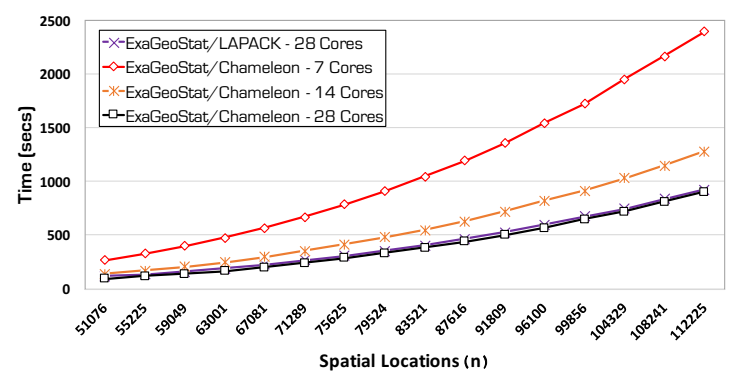

(b) Intel two-socket Broadwell.

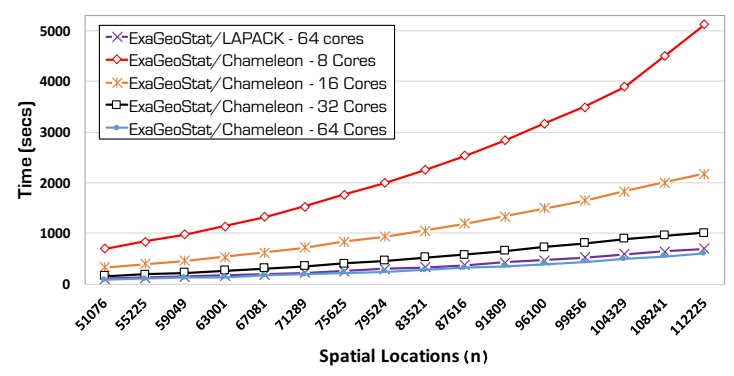

(c) Intel two-socket Knights Landing (KNL).

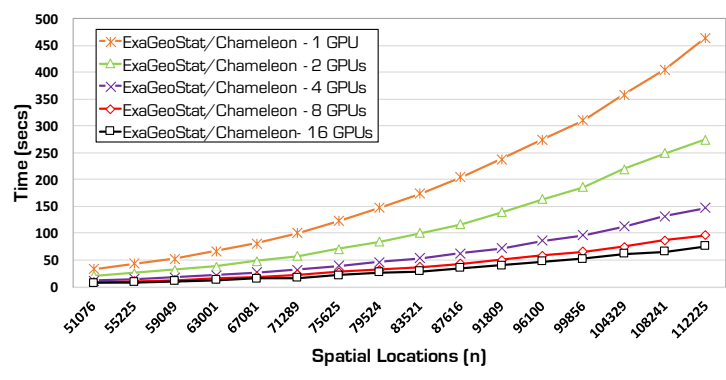

(d) Intel two-socket Haswell + NVIDIA K80.

Fig. 6: Time for one iteration of the likelihood estimation.

of threads. The figures also display the scalability of ExaGeoStat when using different numbers of threads.

Figure 6(a) shows the execution time for a single iteration of MLE with 7,18, and 36 threads compared to the LAPACK implementation on a Haswell processor. As shown, our implementation achieved a $1.14 \times$ speedup compared with the LAPACK implementation by exploiting up to $70 \%$ of the peak performance of the Haswell processor.

Figure $6(\mathrm{~b})$ shows the execution time with 7,14 , and 28 threads compared to the LAPACK implementation on a Broadwell processor. Our chameleon-based platform speeds 


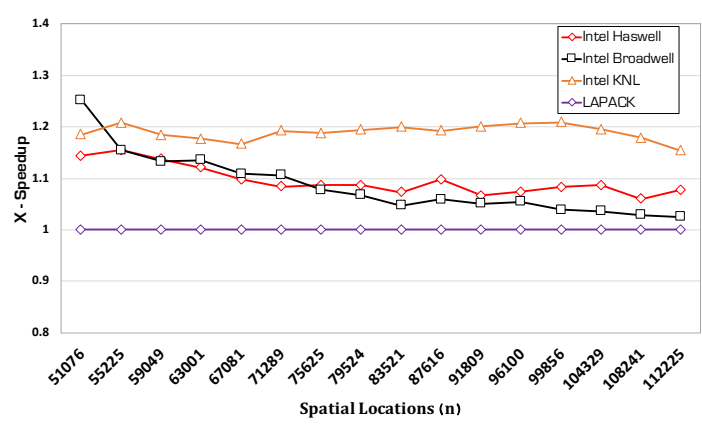

Fig. 7: ExaGeoStat/Chameleon speedup compared to ExaGeoStat/LAPACK on Haswell, Broadwell, and KNL.

up the execution time by $1.25 \times$ with 28 threads compared to the LAPACK implementation. Moreover, our implementation with 28 threads is able to reach over $53 \%$ of the total peak performance of the Broadwell processor, while the LAPACK implementation can only reach $47 \%$ of the peak performance. The figure also shows scalability using different numbers of threads.

The performance of our proposed platform running on an Intel Knights Landing (KNL) processor is reported in Figure 6(c). The platform is easily scaled to accommodate different numbers of threads (i.e., 4, 8, 16, 32, and 64). Using the entire capability of KNL - 64 threads - we achieve an overall speedup of $1.20 \times$ compared to the LAPACK implementation. The achieved flop rate is more than $52 \%$ of the peak performance of the KNL, while the LAPACK implementation achieves only $40 \%$ of the peak.

For the performance analysis using GPUs, a Haswell system with 8 NVIDIA K80s is tested. Figure 6(d) shows the scalability with different numbers of GPUs units. Using $1,2,4,8$, and 16 GPUs, we achieve an average of 1.1, 1.9, 3.1, 5.2, and $6.6 \mathrm{Tflop} / \mathrm{s}$, respectively. With this high flop rate, one iteration of a $100 \mathrm{~K}$ problem can be solved using 16 GPUs in less than 52 seconds.

Figure 7 shows the speedup gained from using ExaGeoStat based on Chameleon compared to LAPACK across a range of matrix sizes. The figure shows the speedup based on the aforementioned Intel architectures, i.e., Haswell, Broadwell, and KNL. The speedup can reach up to $1.4 \times$, $1.25 \times$, and $1.2 \times$ on these systems, respectively. The minimum gained speedup using the Haswell, Broadwell, and KNL processors are $1.025 \times, 1.06 \times$, and $1.15 \times$, respectively. There is a performance trend. For small matrix sizes, the asynchronous Chameleon-based ExaGeoStat performs better than the traditional bulk synchronous LAPACK-based ExaGeoStat since it mitigates the idle time between panel factorization and update of the trailing submatrix. For asymptotic matrix sizes, the performance gap shrinks between both ExaGeoStat variants since the workload is large enough to maintain hardware resources busy.

Figure 8 summarizes the performance of ExaGeoStat on different shared-memory Intel processors. Skylake processor represents the latest available generation of Intel processors. Skylake shows the highest gained in terms of performance on $x 86$ systems, where $100 \mathrm{~K} \times 100 \mathrm{~K}$ problems can be

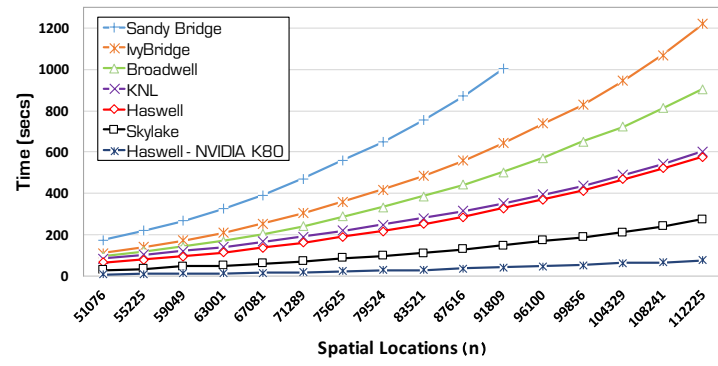

Fig. 8: Hardware-agnostic ExaGeoStat software.

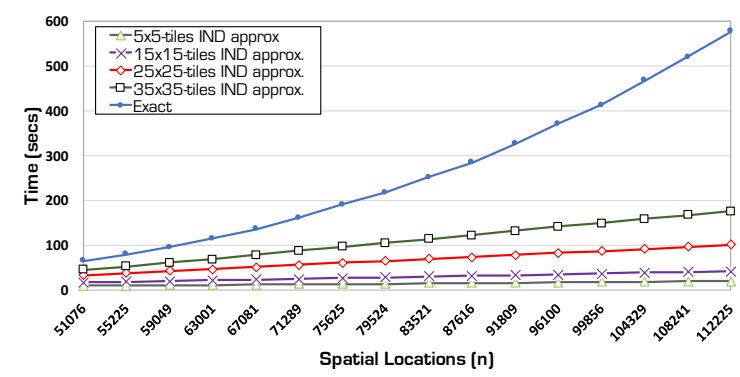

Fig. 9: Time for one iteration of the likelihood estimation using exact and IND computation on Haswell processor.

solved in about 4.5 minutes on 56 cores. This experiment does not aim at comparing performance across Intel processor generations, since each system has a different total number of cores, i.e., Sandy Bridge (32 cores total), IvyBridge (40 cores total), Broadwell (28 cores total), KNL (64 cores), Haswell (36 cores total), Skylake (56 cores total), and eight NVIDIA K80 GPU server. However, this experiment aims at showing how our proposed unified software is hardwareagnostic and can deploy on Intel x86 architectures as well as NVIDIA GPU-based servers, using a single source code, which is further leveraged to distributed-memory environment systems.

Figure 9 shows the performance impact when applying the IND approximation technique on synthetic datasets. We only report the performance on Intel Haswell processor, since similar performance trend may be obtained on the other hardware systems. As expected, the IND approximation method is able to estimate the likelihood function faster than the exact method. The figure also shows that with larger diagonal super tiles, the likelihood estimation time increases, as expected. These performance numbers have to be cautiously put in the context of the qualitative study, presented later in Section 7.5.

We also test our proposed software on the distributedmemory Cray XC40, Shaheen, with different numbers of nodes. Figure 10(a) reports the total execution time in terms of cores (with 32 cores per node). With small matrix sizes, the benefits are modest. However, as the matrix size grows, speedup saturates at higher and higher values. ExaGeoStat is able to solve one maximum likelihood problem of dimension $700 \mathrm{~K}$ in about 800 seconds.

Figure 10(b) shows the performance using the distributed-memory by reporting the flop rate against the 


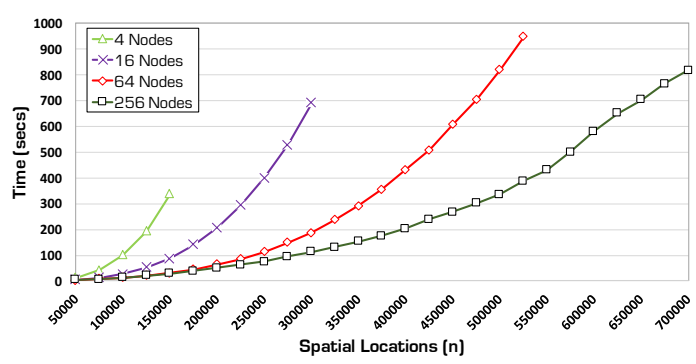

(a) One iteration of likelihood operation - time.

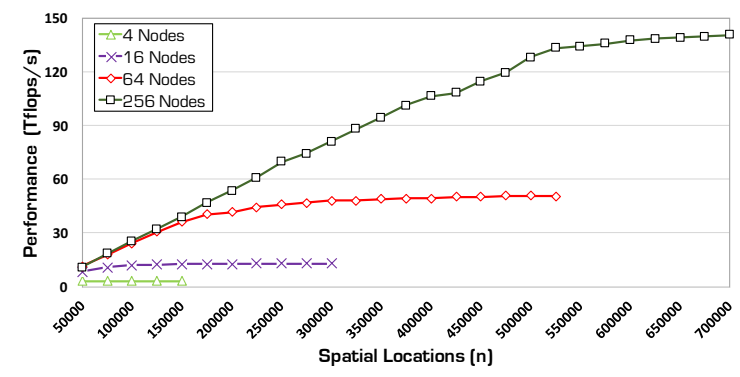

(b) One iteration of likelihood operation - Tflop/s.

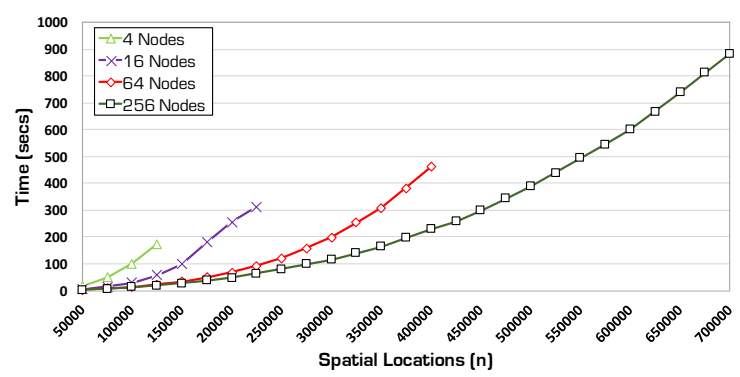

(c) Prediction eval. of 100 unknown values - time.

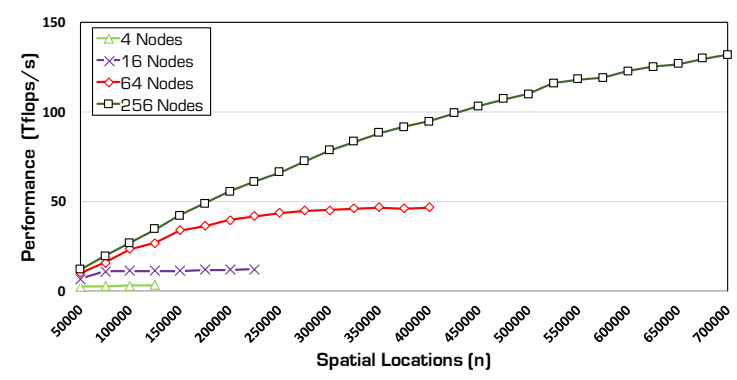

(d) Prediction eval. of 100 unknown values - Tflop/s.

Fig. 10: Distributed-memory performance scalability on Cray XC40.

varying core count. Using 8192 cores, $140 \mathrm{Tflop} / \mathrm{s}$ is achieved on a problem of dimension $700 \mathrm{~K}$. These experiments not only validate the good performance of our unified platform on different hardware architectures, but also, to the best of our knowledge, extend the exact solution of the MLE problem to such unprecedented large sizes. In Figures 10(a) and 10(b), some lines do not extend very far because of memory limits for smaller numbers of cores.

\subsubsection{Prediction Evaluation Performance}

Here, we investigate the performance of the prediction operation (i.e., 100 unknown measurements) using the ExaGeoStat software on a distributed system (i.e., Cray XC40).

Figure 10(c) shows the execution time for the prediction from different sizes synthetic datasets up to $700 \mathrm{~K}$ using $4,16,64$, and 256 Shaheen's nodes, each has 32 cores. The scalability can seen in the figure. The prediction operation for a $700 \mathrm{~K}$ problem size can be evaluated in about 880 seconds.

Figure 10(d) shows the performance in Tflop/s with different numbers of cores. On Shaheen, the prediction operation achieve a $130 \mathrm{Tflop} / \mathrm{s}$ performance for $700 \mathrm{~K}$ problem size using 8192 cores.

\subsection{Qualitative Analysis (Monte Carlo Simulations)}

The overall goal of the maximum likelihood model is to estimate the unknown parameters of the statistical model $\left(\theta_{1}, \theta_{2}\right.$, and $\left.\theta_{3}\right)$ of the Matern covariance function, then to use this model for future predictions of unknown measurements. In this experiment, we use the Monte Carlo simulation to estimate the parameters of an exponential covariance model, where $\theta_{1}=1, \theta_{2}=0.1, \theta_{3}=0.5$.

Using our data generator tool and the initial parameter vector $\left(\theta_{1}=1, \theta_{2}=0.1, \theta_{3}=0.5\right)$, four different synthetic datasets are generated (i.e., $20 \mathrm{~K}, 40 \mathrm{~K}, 60 \mathrm{~K}$, and $80 \mathrm{~K}$ ) besides 100 measurement vectors $\mathbf{Z}$ for each dataset. This experiment is repeated 100 times with different measurement vectors.

Figures 11(a), 11(b), and 11(c) show three boxplots representing the estimated parameters with 100 measurement vectors $\mathbf{Z}$. The true value is denoted by a dotted red line. As shown, all of the results are close to the correct $\boldsymbol{\theta}$ vector.

To evaluate the accuracy of our predictions, we randomly choose a set of locations and mark the measurements on those locations as unknown. Then, using the estimated $\widehat{\boldsymbol{\theta}}$ vector, ExaGeoStat predicts the unknown measurements at those locations with the aid of the known measurements. The accuracy of the prediction operation can be estimated using the Mean Square Error (MSE) between the actual measurements and the predicted ones as $M S E=\frac{1}{n} \sum_{i=1}^{n}\left(\widehat{y_{i}}-y_{i}\right)^{2}$, where $\widehat{y_{i}}$ represents the predicted value and $y_{i}$ represents the actual value at the same location.

Figure 12 shows the boxplot of the predictions MSE using a $k$-fold cross-validation technique, where $k=10$, to validate the prediction accuracy using different synthetic dataset sizes. The total number of missing values equals to $n / k$ (i.e., subsample size). With larger matrix sizes, our prediction implementation has a smaller MSE compared to the smaller matrix sizes. The average execution time per single prediction using four nodes on Shaheen Cray XC40 is shown under each boxplot.

\subsection{Real Dataset Application}

In environmental applications, the number of measurements is usually very large. These measurements are often distributed irregularly across a given geographical region, and can be modeled as a realization from a Gaus- 


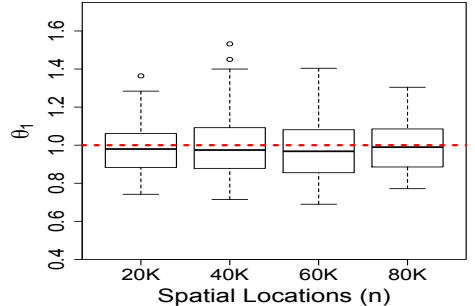

(a) Estimated variance parameter $\left(\theta_{1}\right)$.

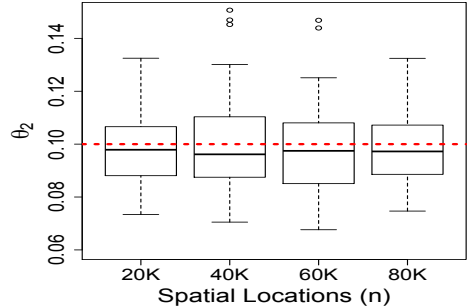

(b) Estimated spatial range parameter $\left(\theta_{2}\right)$.

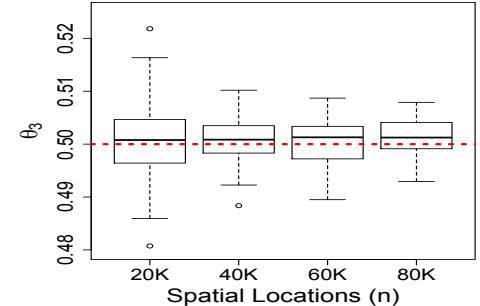

(c) Estimated smoothness parameter $\left(\theta_{3}\right)$.

Fig. 11: Boxplots of parameter estimates.

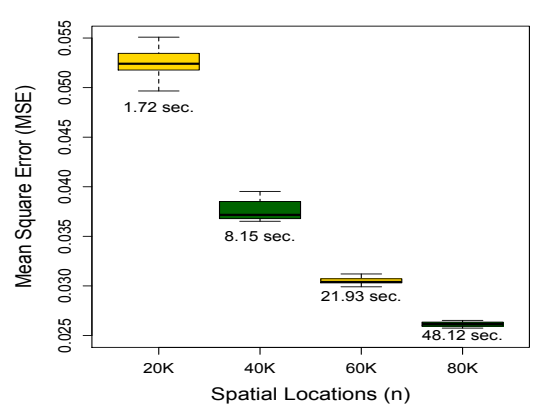

Fig. 12: Predictions MSE of $n / k$ missing values of different synthetic dataset sizes using a $k$-fold cross-validation technique $(k=10)$. Execution time per single prediction is shown under each boxplot using four nodes on Shaheen.

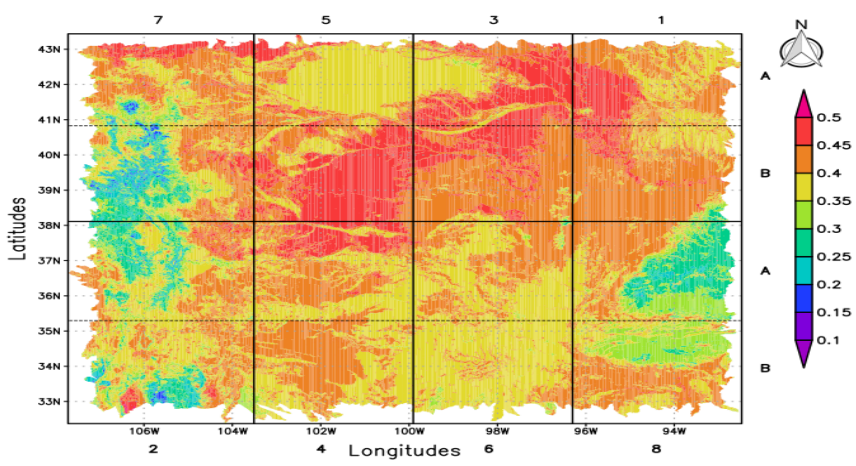

Fig. 13: Soil moisture data divided into 16 geographical regions.

sian spatial random field. In this study, we have evaluated our unified software using a soil moisture data coming from Mississippi River basin region, USA (more details are given in Section 4). Because locations in the soil moisture dataset are given by longitude and latitude pairs, the location space is non-Euclidean. Therefore, we use the Great-Circle Distance (GCD) metric to compute the distance between any given two locations with their original longitude and latitude values. The best representation of the GCD is the haversine formula [38] $\operatorname{hav}\left(\frac{d}{r}\right)=\operatorname{hav}\left(\varphi_{2}-\varphi_{1}\right)+\cos \left(\varphi_{1}\right) \cos \left(\varphi_{2}\right) \operatorname{hav}\left(\lambda_{2}-\lambda_{1}\right)$,
TABLE 1: Estimation of the Matérn covariance parameters for 8 geographical regions and the average prediction MSE using a $k$-fold cross-validation technique $(k=10)$.

\begin{tabular}{|c|c|c|c|c|}
\hline \multirow{2}{*}{ Regions } & \multicolumn{3}{|c|}{ Matérn Covariance } & Avg. \\
& $\begin{array}{c}\text { Variance } \\
\left(\theta_{1}\right)\end{array}$ & $\begin{array}{c}\text { Spatial } \\
\text { Range }\left(\theta_{2}\right)\end{array}$ & $\begin{array}{c}\text { Smoothness } \\
\left(\theta_{3}\right)\end{array}$ & $\begin{array}{c}\text { prediction } \\
\text { MSE }\end{array}$ \\
\hline R 1 & 0.823 & 7.215 & 0.529 & 0.0643 \\
\hline R 2 & 0.481 & 10.434 & 0.500 & 0.0315 \\
\hline R 3 & 0.328 & 10.434 & 0.534 & 0.0175 \\
\hline R 4 & 0.697 & 16.761 & 0.483 & 0.0298 \\
\hline R 5 & 1.152 & 13.431 & 0.482 & 0.0612 \\
\hline R 6 & 0.697 & 16.095 & 0.512 & 0.0263 \\
\hline R 7 & 0.520 & 16.872 & 0.487 & 0.0213 \\
\hline R 8 & 0.390 & 12.321 & 0.447 & 0.0287 \\
\hline
\end{tabular}

where hav is the haversine function, $\operatorname{hav}(\theta)=\sin ^{2}\left(\frac{\theta}{2}\right)=$ $\frac{1-\cos (\theta)}{2} ; d$ is the distance between two locations, $r$ is the radius of the sphere, $\varphi_{1}$ and $\varphi_{2}$ are the latitudes in radians of locations 1 and 2, respectively, and $\lambda_{1}$ and $\lambda_{2}$ are longitudes.

For the soil moisture measurements from such a large spatial region, it is very likely that the process exhibits nonstationarity, i.e., the soil moisture covariance parameters may vary in space. Therefore, it is necessary to understand the features of the dataset before choosing the appropriate statistical model for fitting the data. To examine whether it is reasonable to fit a stationary model to the whole spatial region, we propose dividing the entire region into disjointed subregions and applying our computationally efficient methods to fit stationary Gaussian process models with a Matern covariance function to each subregion. Then we can compare the spatial variability across regions using the parameter estimations. The division is only applied in order to study the behavior of the soil moisture dataset, and does not mean that our method is limited to stationary models. Our method can be used directly on non-stationary covariance models without modification. However, the stationary covariance models are essential in any geospatial analysis and serve as the cornerstones of more complex, non-stationary models.

We consider two different strategies for dividing this dataset, as shown in Figure 13, where the locations are divided into 16 subregions (i.e., $1 \mathrm{~A}, 1 \mathrm{~B}, \ldots$,..tc.) or 8 subregions (i.e., 1, 2, ...etc.) The parameter estimation of the Matern covariance is summarized in Tables 1 and 2 using the GreatCircle Distance (GCD).

From both tables, we see that the marginal variance $\theta_{1}$ 
TABLE 2: Estimation of the Matern covariance parameters for 16 geographical regions and the average prediction MSE using a $k$-fold cross-validation technique $(k=10)$.

\begin{tabular}{|c|c|c|c|c|}
\hline \multirow{2}{*}{ Regions } & \multicolumn{3}{|c|}{ Matérn Covariance } & Avg. \\
& $\begin{array}{c}\text { Variance } \\
\left(\theta_{1}\right)\end{array}$ & $\begin{array}{c}\text { Spatial } \\
\text { Range }\left(\theta_{2}\right)\end{array}$ & $\begin{array}{c}\text { Smoothness } \\
\left(\theta_{3}\right)\end{array}$ & $\begin{array}{c}\text { prediction. } \\
\text { MSE }\end{array}$ \\
\hline R 1A & 0.852 & 5.994 & 0.559 & 0.0711 \\
\hline R 1B & 0.380 & 10.434 & 0.490 & 0.0527 \\
\hline R 2A & 0.277 & 10.878 & 0.507 & 0.0202 \\
\hline R 2B & 0.410 & 7.77 & 0.527 & 0.0303 \\
\hline R 3A & 0.836 & 9.213 & 0.496 & 0.0619 \\
\hline R 3B & 0.619 & 10.323 & 0.523 & 0.0355 \\
\hline R 4A & 0.553 & 19.203 & 0.508 & 0.0186 \\
\hline R 4B & 0.906 & 27.861 & 0.461 & 0.0298 \\
\hline R 5A & 1.619 & 17.205 & 0.466 & 0.0775 \\
\hline R 5B & 1.028 & 24.531 & 0.498 & 0.0296 \\
\hline R 6A & 0.599 & 25.197 & 0.457 & 0.0219 \\
\hline R 6B & 0.332 & 12.432 & 0.418 & 0.0294 \\
\hline R 7A & 0.625 & 7.659 & 0.523 & 0.0463 \\
\hline R 7B & 0.467 & 9.324 & 0.549 & 0.0244 \\
\hline R 8A & 0.485 & 12.654 & 0.464 & 0.0313 \\
\hline R 8B & 0.383 & 13.875 & 0.477 & 0.0211 \\
\hline
\end{tabular}

and the spatial range parameter $\theta_{2}$ change across regions, suggesting that the local variability shows obvious nonstationarity. However, the smoothness parameter $\theta_{3}$ hardly changes at all across different regions. In the future studies, we may merge the subregions with similar parameter estimates and fit one stationary model to that combined region, while investigating the covariances of those subregions with very different parameter estimates more carefully.

We also estimate the accuracy by validating the estimated model parameters using a prediction evaluation process. A $k$-fold cross-validation technique has been used, where $k=10$. In this case, the number of missing values that have been chosen from large regions, i.e., $250 \mathrm{~K}$, is 25000 , and from small regions, i.e., $125 \mathrm{~K}$, is 12500 . We applied the $k$-fold cross-validation technique and reported the average MSE.

Although GCD may be one of the best representations of the distance between two points on the surface of a sphere such as the earth, we have also tried the Euclidean Distance (ED) metric for the soil moisture data, after transforming soil moisture dataset to the Euclidean space. We have found that none of GCD and ED are uniformly better than the other and, therefore, have decided to only report GCD metric.

\subsection{Qualitative Analysis Using Real Application}

Evaluating the accuracy of exact computation compared to approximation techniques is necessary to highlight the advantage of using such a computational-intensive software to solve the likelihood estimation problem. As mentioned earlier, one of the main goals of this study is to build a benchmark software to validate existing or future approximation techniques with large-scale data.

In this experiment, we compare exact computation with IND approximation, i.e., both are provided by the ExaGeoStat software. The experiment is performed on one region

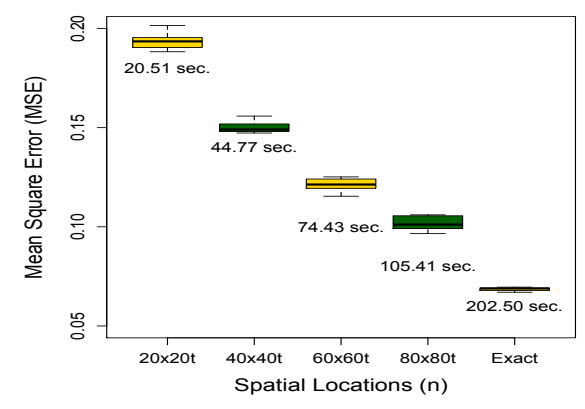

Fig. 14: Predictions MSE of 12500 missing values of region $1 \mathrm{~A}$ of the soil moisture dataset using both exact and IND approx. methods with a $k$-fold cross-validation technique $(k=10)$. Execution time per single prediction is shown under each boxplot using four nodes on Shaheen.

of soil moisture dataset (1A). The selected region has $125 \mathrm{~K}$ measurements with a location matrix of size $125 \mathrm{~K} \times 125$ $\mathrm{K}$ elements. To gain the best performance of ExaGeoStat, we tune the tile size to be 560, with Cray XC40 cluster to optimize the performance. In this case, the whole location matrix is divided to 224 tiles in each dimension, i.e., total number of tiles $=224 \times 224=50176$ tiles. In the case of IND approximation, a different size of diagonal super tiles is used, i.e., $20 \times 20,40 \times 40,60 \times 60$, and $80 \times 80$. Of course, large diagonal super tiles show more accuracy improvements because more locations are taken into account. The experiment has been repeated for 100 times, each aims at predicting different 100 missing values randomly selected from the same region.

Figure 14 shows the predictions MSE for various sizes of diagonal super tiles using IND approximation technique compared to the exact solution on region 1A. A $k$-fold cross-validation technique, where $k=10$, is used to validate the prediction accuracy, where the total number of missing values equals to 12500 (i.e., $n / k$ subsample). As shown, exact computation satisfies the lowest MSE prediction compared to IND approximation technique. The figure also illustrates that lower MSE prediction values can be obtained by increasing the size of diagonal super tiles in the case of IND approximation. In this case, more computation power is required to complete the whole estimation operation. Moreover, the average execution time per single prediction on four Shaheen nodes is shown under each boxplot.

\section{Conclusion AND Future Work}

This paper highlights the ability of the new ExaGeoStat software to estimate the maximum likelihood function in the context of climate and environmental applications and to predict missing measurements across geographical locations. This software provides a full machine learning pipeline (i.e., estimation, model fitting, and prediction) for geostatistics data. ExaGeoStat is able to run with a decent performance on a wide range of hardware architectures, thanks to the high-performance, dense linear algebra library Chameleon, associated with the StarPU runtime system. 
We have successfully applied the software to synthetic and real datasets. Since calculations are performed without any approximations, the estimated parameters can be used as a references for assessing different approaches, with the end goal of generating online database containing an ensemble of parameter estimates.

We also provide the implementation of an $R$ wrapper API, i.e., ExaGeoStatR, to ease the process of integrating ExaGeoStat with the computational statistician community. The $R$ package includes the main functions that can be used by statisticians to evaluate the MLE operation on variant hardware architectures.

In the future work, we plan to investigate hierarchical matrix approximations based on $\mathcal{H}$-matrices, which will allow us to replace dense subblocks of the exact matrix with low-rank approximations in an accuracy-tunable manner, significantly reducing the memory footprint and operation count without compromising the accuracy of the applications [3]. We also plan to support our package with NetCDF support to deal with a wide range of existing climate and environment data.

\section{ACKNOWLEDGEMENT}

The research reported in this publication was supported by funding from King Abdullah University of Science and Technology (KAUST). We would like to thank NVIDIA for hardware donations in the context of a GPU Research Center, Intel for support in the form of an Intel Parallel Computing Center award, Cray for support provided during the Center of Excellence award to the Extreme Computing Research Center at KAUST, and KAUST IT Research Computing for their hardware support on the GPU-based system. This research made use of the resources of the KAUST Supercomputing Laboratory. Finally, the authors would like to thank Alexander Litvinenko from the Extreme Computing Research Center for his valuable help.

\section{References}

[1] E. Agullo, A. Buttari, A. Guermouche, and F. Lopez, "Task-based multifrontal QR solver for GPU-accelerated multicore architectures," in 22nd IEEE International Conference on High Performance Computing (HiPC). IEEE Computer Society, 2015, pp. 54-63.

[2] E. Agullo, J. Demmel, J. Dongarra, B. Hadri, J. Kurzak, J. Langou, H. Ltaief, P. Luszczek, and S. Tomov, "Numerical Linear Algebra on Emerging Architectures: The PLASMA and MAGMA projects," in Journal of Physics: Conference Series, vol. 180, 2009.

[3] K. Akbudak, H. Ltaief, A. Mikhalev, and D. Keyes, "Tile Low Rank Cholesky Factorization for Climate/Weather Modeling Applications on Manycore Architectures," in Proceedings of the International Supercomputing Conference (ISC'17) (J. M. Kunkel et al., eds), Lecture Notes in Computer Science, Springer, Volume 10266, pp. 22-40, 2017.

[4] E. Anderson, Z. Bai, C. Bischof, L. S. Blackford, J. Demmel, J. Dongarra, J. Du Croz, A. Greenbaum, S. Hammarling, A. McKenney et al., LAPACK Users' Guide. SIAM, 1999.

[5] C. Augonnet, S. Thibault, R. Namyst, and P.-A. Wacrenier, "StarPU: A Unified Platform for Task Scheduling on Heterogeneous Multicore Architectures," Concurrency and Computation: Practice and Experience, vol. 23, no. 2, pp. 187-198, 2011.

[6] - "StarPU: a unified platform for task scheduling on heterogeneous multicore architectures," Concurrency and Computation: Practice and Experience, vol. 23, no. 2, pp. 187-198, 2011.

[7] S. Banerjee, A. E. Gelfand, A. O. Finley, and H. Sang, "Gaussian predictive process models for large spatial data sets," Journal of the Royal Statistical Society: Series B (Statistical Methodology), vol. 70, no. 4, pp. 825-848, 2008.
[8] L. A. Berry, W. R. Elwasif, J. M. Reynolds-Barredo, D. Samaddar, R. Sánchez, and D. E. Newman, "Event-based parareal: A data-flow based implementation of parareal," Journal of Computational Physics, vol. 231, no. 17, pp. 5945-5954, 2012.

[9] L. S. Blackford, J. Choi, A. Cleary, E. F. D'Azevedo, J. W. Demmel, I. S. Dhillon, J. J. Dongarra, S. Hammarling, G. Henry, A. Petitet K. Stanley, D. W. Walker, and R. C. Whaley, ScaLAPACK Users' Guide. Philadelphia: Society for Industrial and Applied Mathematics, 1997.

[10] S. Börm and J. Garcke, "Approximating Gaussian processes with $H^{2}$-matrices," in Proceedings of 18th European Conference on Machine Learning, Warsaw, Poland, September 17-21, 2007. ECML 2007, J. N. Kok, J. Koronacki, R. L. de Mantaras, S. Matwin, D. Mladen, and A. Skowron, Eds., vol. 4701, 2007, pp. 42-53.

[11] W. Kleiber and D. W. Nychka, "Equivalent kriging," Spatial Statistics, vol. 12, pp. 31-49, 2015.

[12] J. E. Castrillon, M. G. Genton, and R. Yokota, "Multi-Level Restricted Maximum Likelihood Covariance Estimation and Kriging for Large Non-Gridded Spatial Datasets," Spatial Statistics, pp. 105124, 2016.

[13] "The Chameleon project," January 2017, available at https:// project.inria.fr/chameleon/.

[14] J.-P. Chiles and P. Delfiner, Geostatistics: modeling spatial uncertainty. John Wiley \& Sons, 2009, vol. 497.

[15] N. Cressie and G. Johannesson, "Fixed rank kriging for very large spatial data sets," Journal of the Royal Statistical Society: Series B (Statistical Methodology), vol. 70, no. 1, pp. 209-226, 2008.

[16] N. Cressie and C. K. Wikle, Statistics for spatio-temporal data. John Wiley \& Sons, 2015.

[17] A. Datta, S. Banerjee, A. O. Finley, and A. E. Gelfand, "Hierarchical nearest-neighbor Gaussian process models for large geostatistical datasets," Journal of the American Statistical Association, vol. 111, pp. 800-812, 2016.

[18] J. Dongarra, P. Beckman, T. Moore, P. Aerts, G. Aloisio, J.-C. Andre, D. Barkai, J.-Y. Berthou, T. Boku, B. Braunschweig et al., "The international exascale software project roadmap," The International Journal of High Performance Computing Applications, vol. 25, pp. 3-60, 2011.

[19] Douglas Nychka, Reinhard Furrer, John Paige, and Stephan Sain, "fields: Tools for spatial data," University Corporation for Atmospheric Research, Boulder, CO, USA, 2015, r package version 9.0. [Online]. Available: www.image.ucar.edu/fields

[20] A. Duran, R. Ferrer, E. Ayguadé, R. M. Badia, and J. Labarta, "A proposal to extend the openmp tasking model with dependent tasks," International Journal of Parallel Programming, vol. 37, no. 3, pp. 292-305, 2009.

[21] H. C. Edwards, C. R. Trott, and D. Sunderland, "Kokkos: Enabling manycore performance portability through polymorphic memory access patterns," Journal of Parallel and Distributed Computing, vol. 74, no. 12, pp. 3202-3216, 2014. [Online]. Available: http://dx.doi.org/10.1016/j.jpdc.2014.07.003

[22] M. Fuentes, "Approximate likelihood for large irregularly spaced spatial data," Journal of the American Statistical Association, vol. 102, pp. 321-331, 2007.

[23] G.-A. Fuglstad, D. Simpson, F. Lindgren, and H. Rue, "Does non-stationary spatial data always require non-stationary random fields?" Spatial Statistics, vol. 14, pp. 505-531, 2015.

[24] R. Furrer, M. G. Genton, and D. Nychka, "Covariance tapering for interpolation of large spatial datasets," Journal of Computational and Graphical Statistics, vol. 15, no. 3, pp. 502-523, 2006.

[25] M. G. Genton, "Separable approximations of space-time covariance matrices," Environmetrics, vol. 18, no. 7, pp. 681-695, 2007.

[26] M. Gil, M. S. Zanetti, S. Zoller, and M. Anisimova, "Codonphyml: fast maximum likelihood phylogeny estimation under codon substitution models," Molecular Biology and Evolution, vol. 30, no. 6, pp 1270-1280, 2013.

[27] P. Guttorp and T. Gneiting, "Studies in the history of probability and statistics XLIX: On the Matérn correlation family," Biometrika, vol. 93, pp. 989-995, 2006.

[28] M. S. Handcock and M. L. Stein, "A Bayesian analysis of kriging," Technometrics, vol. 35, pp. 403-410, 1993.

[29] — "Hierarchical low rank approximation of likelihoods for large spatial datasets," Journal of Computational and Graphical Statistics, no. just-accepted, 2017.

[30] S. G. Johnson, "The NLopt nonlinear-optimization package," 2014. 
[31] L. Leemis, Learning Base $R$. Lightning Source, 2016. [Online]. Available: http://www.amazon.com/ Learning-Base-Lawrence-Mark-Leemis/dp/0982917481

[32] F. Lindgren, H. Rue, and J. Lindström, "An explicit link between Gaussian fields and Gaussian Markov random fields: the stochastic partial differential equation approach," Journal of the Royal Statistical Society: Series B (Statistical Methodology), vol. 73, pp. 423-498, 2011.

[33] A. Litvinenko, Y. Sun, M. G. Genton, and D. Keyes, "Likelihood Approximation With Hierarchical Matrices For Large Spatial Datasets," ArXiv e-prints, Sep. 2017.

[34] B. Matérn, Spatial Variation, second edition ed., ser. Lecture Notes in Statistics. Berlin; New York: Springer-Verlag, 1986, vol. 36.

[35] G. R. North, J. Wang, and M. G. Genton, "Correlation models for temperature fields," Journal of Climate, vol. 24, pp. 5850-5862, 2011.

[36] D. Nychka, S. Bandyopadhyay, D. Hammerling, F. Lindgren, and S. Sain, "A multiresolution Gaussian process model for the analysis of large spatial datasets," Journal of Computational and Graphical Statistics, vol. 24, no. 2, pp. 579-599, 2015.

[37] M. J. Powell, "The BOBYQA algorithm for bound constrained optimization without derivatives," Cambridge NA Report NA2009/06, University of Cambridge, 2009.

[38] D. Rick, "Deriving the haversine formula," in The Math Forum, April, 1999

[39] H. Rue and L. Held, "Gaussian Markov random fields: theory and applications," 2005.

[40] H. Rue and H. Tjelmeland, "Fitting Gaussian Markov random fields to Gaussian fields," Scandinavian Journal of Statistics, vol. 29, no. 1, pp. 31-49, 2002.

[41] H. Sang and J. Z. Huang, "A full scale approximation of covariance functions for large spatial data sets," Journal of the Royal Statistical Society: Series B (Statistical Methodology), vol. 74, no. 1, pp. 111-132, 2012.

[42] M. L. Stein, "Statistical properties of covariance tapers," Journal of Computational and Graphical Statistics, vol. 22, no. 4, pp. 866-885, 2013.

[43] - "Limitations on low rank approximations for covariance matrices of spatial data," Spatial Statistics, vol. 8, pp. 1-19, 2014.

[44] M. L. Stein, Z. Chi, and L. J. Welty, "Approximating likelihoods for large spatial data sets," Journal of the Royal Statistical Society: Series B (Statistical Methodology), vol. 66, no. 2, pp. 275-296, 2004. [Online]. Available: http://dx.doi.org/10.1046/j.1369-7412.2003.05512.x

[45] Y. Sun, B. Li, and M. G. Genton, "Geostatistics for large datasets,"

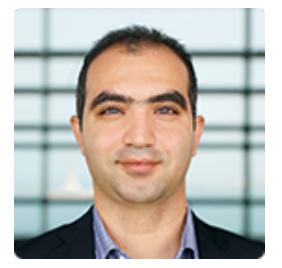

Sameh Abdulah is a Postdoctoral Fellow at the Extreme Computing Research Center, King Abdullah University of Science and Technology, Saudi Arabia. Sameh received his MS and PhD degrees from Ohio State University, Columbus, centered USA, in 2014 and 2016, His work is centered around High Performance Computing (HPC) applications, bitmap indexing in big data, large spatial datasets, parallel statistical applications, algorithm-based fault tolerance, and $\mathrm{Ma}$ chine Learning and Data Mining algorithms.

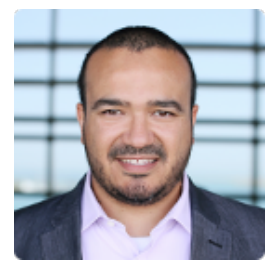

Hatem Ltaief is a Senior Research Scientis in the Extreme Computing Research Center at King Abdullah University of Science and Technology, Saudi Arabia. His research interests include parallel numerical algorithms, fault tolerant algorithms, parallel programming models, and performance optimizations for multicore architectures and hardware accelerators. His current research collaborators include Aramco, Total, Observatoire de Paris, NVIDIA, and Intel. in Space-Time Processes and Challenges Related to Environmental Problems, M. Porcu, J. M. Montero, and M. Schlather, Eds. Springer, 2012, pp. 55-77.

[46] Y. Sun and M. L. Stein, "Statistically and computationally efficient estimating equations for large spatial datasets," Journal of Computational and Graphical Statistics, vol. 25, no. 1, pp. 187-208, 2016.

Ying Sun is an Assistant Professor of Statis-

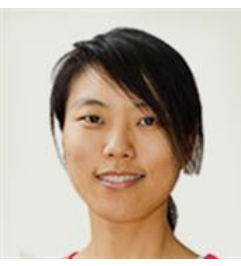

tics at King Abdullah University of Science and Technology (KAUST) in Saudi Arabia. She joined KAUST in June 2014 after one year of service as an assistant professor in the Department of Statistics at Ohio State University, USA. At KAUST, she leads a multidisciplinary research group on environmental statistics, dedicated to developing statistical models and methods for using space-time data to solve important environmental problems. Prof. Sun received her Ph.D. degree in Statistics from Texas A\&M University in 2011, and was a postdoctoral researcher in the research network of Statistics in the Atmospheric and Oceanic Sciences (STATMOS), affiliated with the University of Chicago and the Statistical and Applied Mathematical Sciences Institute (SAMSI). Her research interests include spatio-temporal statistics with environmental applications, computational methods for large datasets, uncertainty quantification and visualization, functional data analysis, robust statistics, and statistics of extremes.

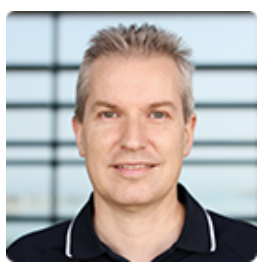

Marc G. Genton is a Distinguished Professor of Statistics at King Abdullah University of Science and Technology (KAUST) in Saudi Arabia. He received his Ph.D. in Statistics from the Swiss Federal Institute of Technology (EPFL), Lausanne, in 1996. He also holds a M.Sc. in Applied Mathematics Teaching and a degree of Applied Mathematics Engineering from the same institution. Prof. Genton is a Fellow of the American Statistical Association, of the Institute of Mathematical Statistics, and the American Association for the Advancement of Science, and is an elected member of the International Statistical Institute. In 2010, he received the ElShaarawi award for excellence from the International Environmetrics Society and the Distinguished Achievement award from the Section on Statistics and the Environment of the American Statistical Association. Prof. Genton's research interests include statistical analysis, flexible modeling, prediction, and uncertainty quantification of spatio-temporal data, with applications in environmental and climate science, renewable energies, geophysics, and marine science.

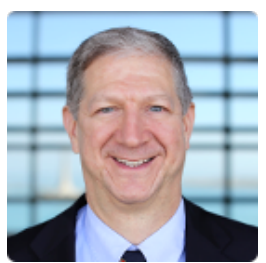

David Keyes directs the Extreme Computing Research Center at KAUST. He earned a BSE in Aerospace and Mechanical Sciences from Princeton University in 1978, and $\mathrm{PhD}$ in Applied Mathematics from Harvard University in 1984. Keyes works at the interface between parallel computing and the numerical analysis of PDEs, with a focus on scalable implicit solvers. He helped develop and popularize the NewtonKrylov-Schwarz (NKS), Additive Schwarz Preconditioned Inexact Newton (ASPIN), and Algebraic Fast Multipole (AFM) methods. Before joining KAUST as a founding dean in 2009, he led multi-institutional projects researching scalable solver software in the SciDAC and ASCI programs of the US DOE, ran University collaboration programs at LLNL's ISCR and NASA's ICASE, and taught at Columbia, Old Dominion, and Yale Universities. He is a Fellow of SIAM and AMS, and has been awarded the ACM Gordon Bell Prize, the IEEE Sidney Fernbach Award, and the SIAM Prize for Distinguished Service to the Profession. 